No. 13(20)

\title{
THE CORIOLIS FORCE AND THE CONCEPT OF THE CORIOLIS POWER PLANT
}

\section{Tadeusz Janaszak}

\begin{abstract}
This paper presents analyses of the fictitious forces which are appearing where the frame of reference is moving simultaneously on the linear and rotational path. Finally, it gives a concept of the construction of a power plant. The power plant used the energy which derived from the rotation of the Earth.
\end{abstract}

Keywords: complex number, derivative, Coriolis acceleration, Coriolis force, Coriolis power plant.

JEL Classification: C02.

DOI: $10.15611 /$ me.2017.13.02.

\section{Introduction}

It is possible to give a lecture on algebra or calculus without any examples of physics, economy, chemistry, or any other branch of science. A lecture using only strictly mathematical methods is sufficient. But there are some reasons for giving in the mathematical lecture some examples from other branches of science.

Firstly: mathematics is often used in the other branches of science as a precise language. Sometimes there is a chance that from physics or economics or another branch comes an impulse for the development of mathematics, for example the calculus has its starting point in physics.

Secondly: some problems in different branches of science are described with the same mathematical language. For example speed in physics and marginal cost in economics are described by derivatives.

Thirdly: the lectures on mathematics, physics, and economics are given in parallel during the studies. One ought to show in the lecture of mathematics some application of mathematical notion on the other branches of science. The 'pure' mathematics is insufficient.

\footnotetext{
Tadeusz Janaszak

Wrocław University of Economics e-mail: tadeusz.janaszak@ue.wroc.pl ORCID: 0000-0001-8981-3355
} 
This paper presents a description of the Coriolis force by using some functions with real argument and complex value. At the end of the paper a project using the energy of the rotation motion of the Earth to the production of electrical energy is shown.

\section{Fundamental principles of rotational motion with constant velocity}

Suppose that a particle is moving all around a point $O$ with a constant angular velocity $\omega$. Let the distance between the particle and the centre $O$ equal $r$. The rotating motion may be described by function $f: R \rightarrow R^{2}$ where $R$ is the field of real numbers - the time axis, and $R^{2}$ is the plane i.e. $f: R \rightarrow C$ where $C$ is the field of complex numbers - the location of the particle. Let the centre of the rotating $O$ be the zero of the complex number plane. On any moment of the time the particle is situated in the circle $C_{r}=\{z \in C:|z|=r\}$, i.e. $f: R \rightarrow C_{r}$. Suppose simply that in the moment $t=0$ the particle is situated on the positive axis $\operatorname{Re} z$. So $z=f(t)$ where

$$
z=r \cdot \exp (i \omega t)
$$

where by definition: for $u \in C$ it is $e^{u}=\exp (u)$. In formula (1) the parameter $\omega$ is the angular velocity; it is a constant. In a more complicated case the location of the particle is given by the formula

$$
z=r \cdot \exp (i \cdot \varphi(t))
$$

where $\varphi(t)$ the angular displacement. If $\varphi(t)=\omega \cdot t$, as in formula (1), the angular velocity is constant. The situation for $\omega>0$ is presented in Figure 1.

The unit vector $\operatorname{sgn} z$ is the versor of the radius vector $r=z$ of the position of the particle ${ }^{1}$, i.e.

$$
\operatorname{sgn} z=\exp (i \omega t) \text {. }
$$

Angle $\varphi$ is the argument of the number $z$, i.e. $\varphi=\arg z$. This is the angular displacement of the particle. Arch $s$ is the path of the particle along circle $C_{r}$. The time of the motion of the particle is considered in interval [0,t]. The formulae $s=r \varphi$, and $s=r \omega t$, and $\varphi=\omega t$ hold.

\footnotetext{
${ }^{1}$ The radius vector $\boldsymbol{r}$ is said the leading vector of the position of the particle. It is possible to write the equality $\boldsymbol{r}=z$ even though $\boldsymbol{r}$ is a vector and $z$ is a complex number.
} 


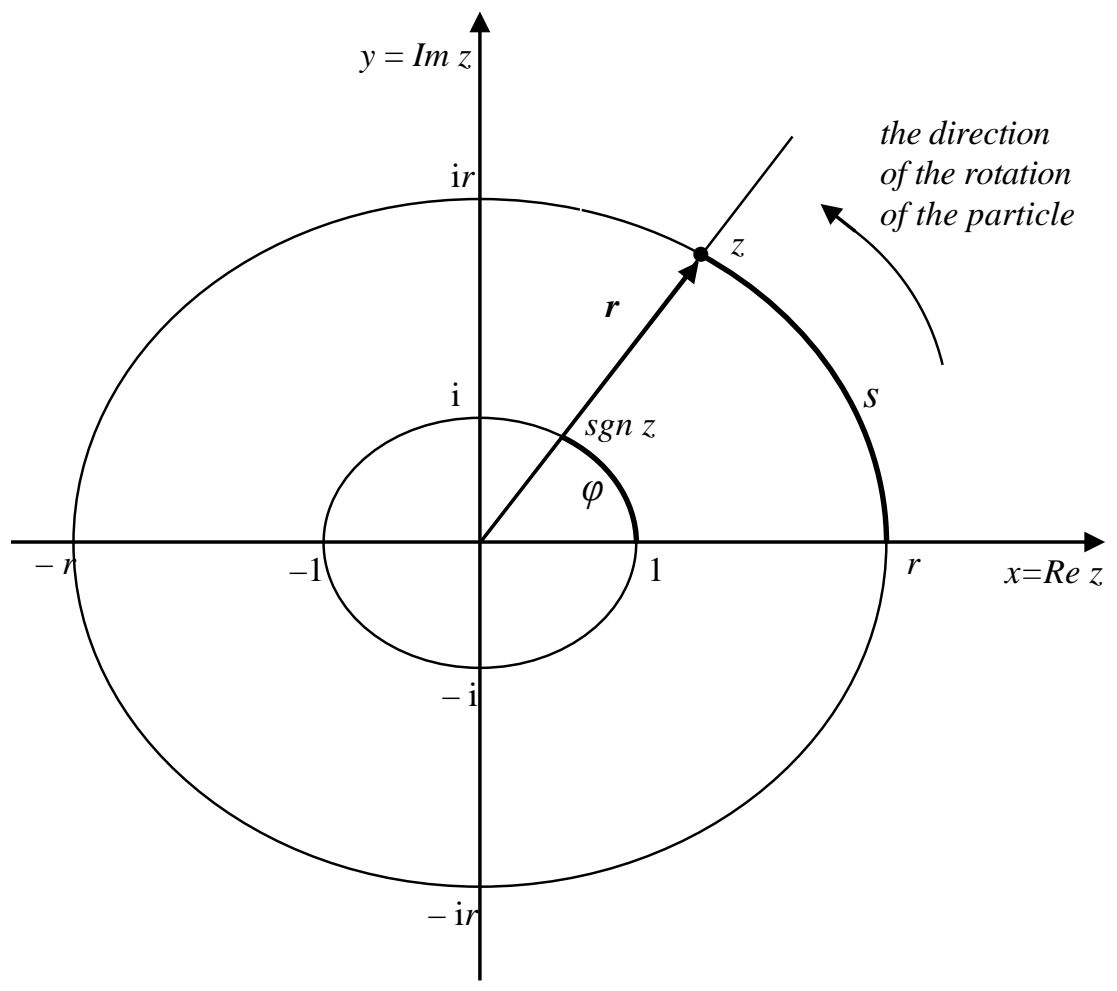

Fig. 1. The rotation of the particle on the complex number plane

Source: own elaboration.

The velocity $v$ of the particle is vector: $v=\frac{d z}{d t}$, i.e.

$$
z^{\prime}=i \cdot \omega \cdot r \cdot \exp (i \omega t) .
$$

Since it is $i=\exp \left(i \cdot \frac{\pi}{2}\right)$ formula (2) is equivalent

$$
z^{\prime}=\omega \cdot r \cdot \exp \left(i \cdot\left(\omega t+\frac{\pi}{2}\right)\right) .
$$

From (1) and (3) it follows that vectors $z$ and $z$ ' are perpendicular. The versor of the vector of velocity $\boldsymbol{v}=z^{\prime}$ is the rotation of the versor of position $z$ of the particle. The angle of this rotation equals $\pi / 2$ and its direction corresponds with the direction of the rotation of the particle, see Figure 2. 


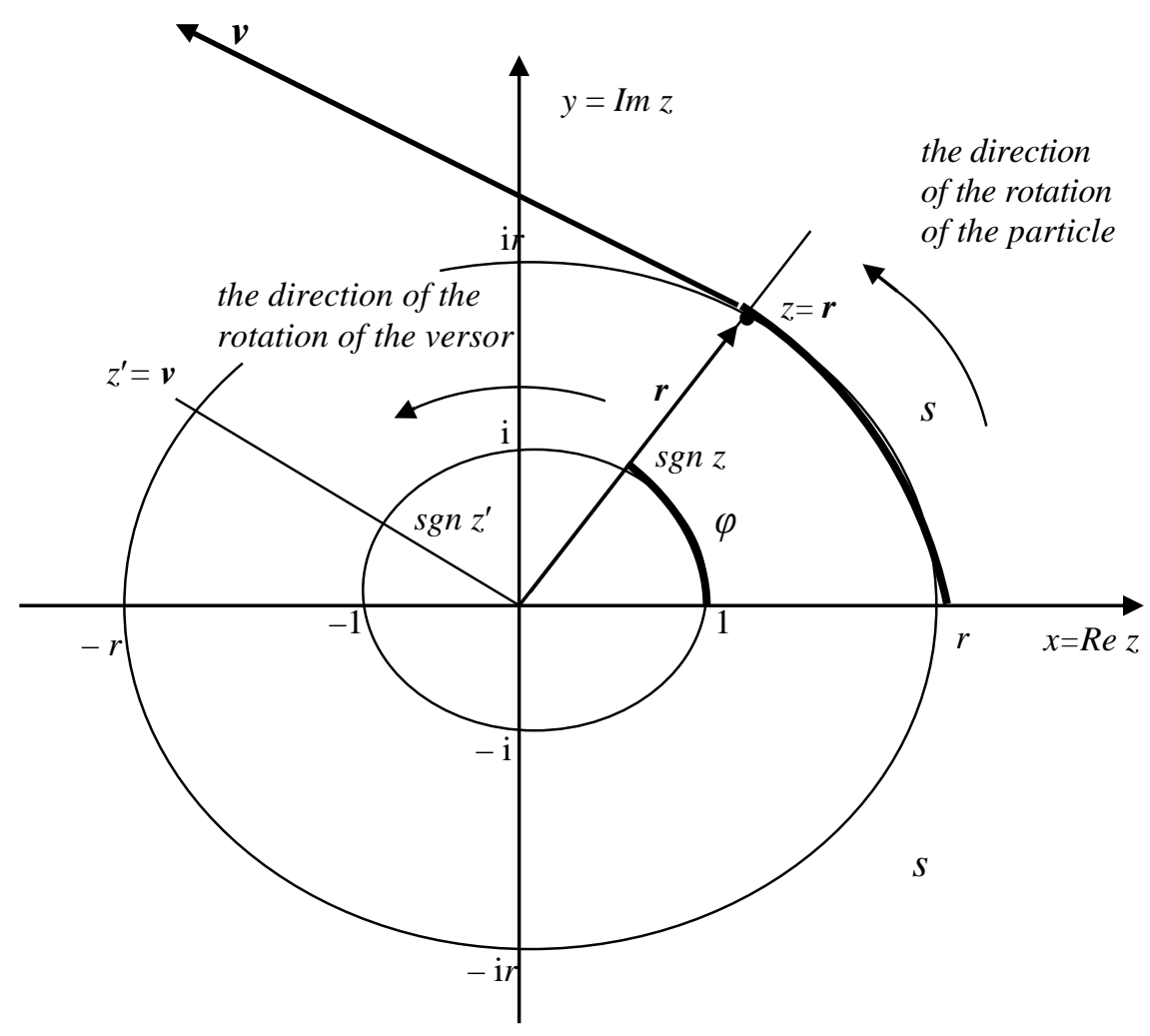

Fig. 2. The vector of the linear velocity and its versor

Source: own elaboration.

Suppose that the standard unit of the length is equal the radius of circle $|z|=1$ in Figure 2 and angle $\varphi$ is crossed during the standard unit of time. Then the length of the path crossed by the particle during the same unit of time equals $s$, i.e. the length of the vector of linear velocity equals $s$. From the lecture on physics it is known that angular velocity is represented by the vector perpendicular to the plane of the rotation. The direction of the rotation and the sense of the vector are such that they both form a right-handed system ${ }^{2}$.

\footnotetext{
2 The observer is standing on the plane of rotation with their head in the sense of the vector perpendicular to the rotating plane. The direction of the rotation and the sense of the vector form the right-handed system if the rotation is seen by the observer as counter-clockwise.
} 
In Figure 2 it is, with these assumptions, that the vector of angular velocity passes from the figure to the face of the observer and its length is equal to the length of $\operatorname{arch} \varphi$. This situation is presented in Figure 3 .

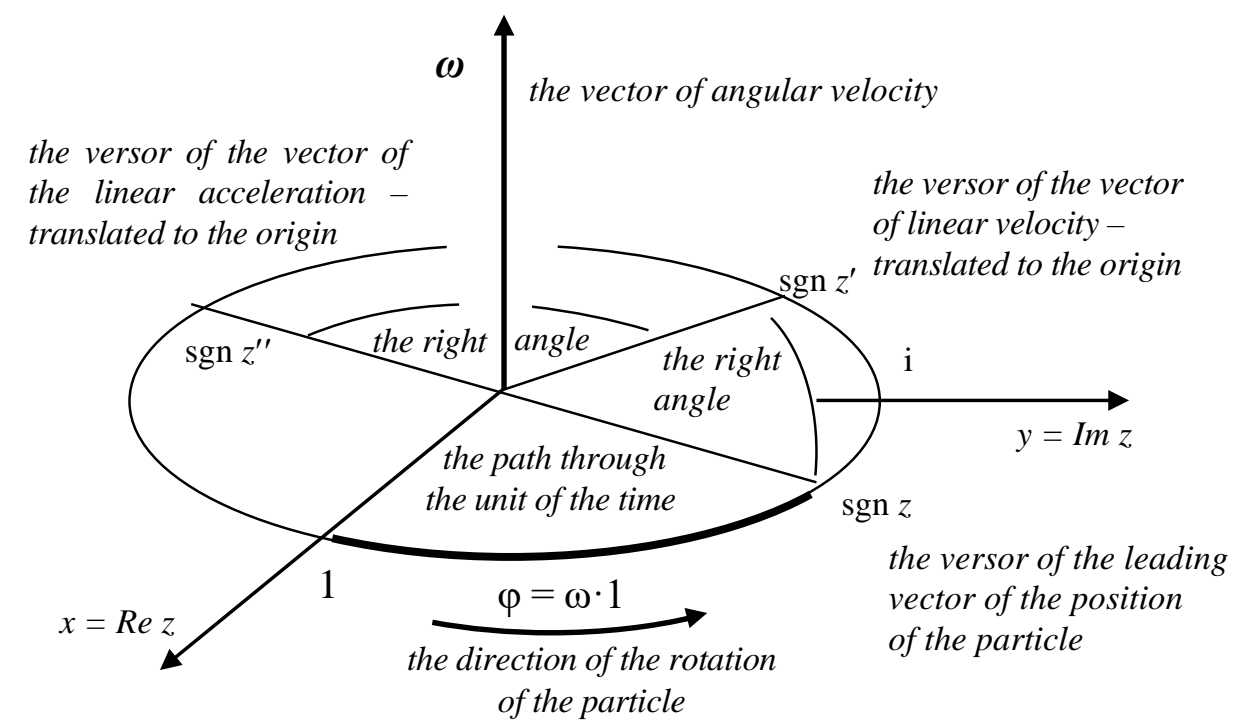

Fig. 3. The direction of the rotation of the particle, the versors of the vectors of the position, linear velocity, and linear acceleration; the direction, the sense and the length of the vector of the angular velocity

Source: own elaboration.

The position of the particle is represented by the complex number $z$. Vector $\boldsymbol{r}$ passes from the origin to the position $z$ is the leading vector for the particle. It is possible to write the equality $z=r$, similarly $z^{\prime}=\boldsymbol{v}$. Vector $\boldsymbol{\omega}$ is the vector of angular velocity which equals the vector product of the leading vector of position of particle $\boldsymbol{r}=z$ and the vector of linear velocity $\boldsymbol{v}=z^{\prime}$ multiplied by the real number that is equal to the inverse of the square of the length of vector of the position, i.e.

$$
\omega=\frac{1}{r^{2}} \cdot(\boldsymbol{r} \times \boldsymbol{v}) .
$$

All The vectors of the formula above are perpendicular. It is possible to write the formula that gives the vector of the linear velocity $\boldsymbol{v}=\boldsymbol{\omega} \times \boldsymbol{r}$ using the convex number

$$
\boldsymbol{v}=r \cdot(\omega \times \operatorname{sgn} \mathbf{z}),
$$

where sgn $\mathbf{z}$ is the leading vector of the complex number sign $\mathrm{z}$. 
To count the acceleration of the particle it is necessary to differentiate function (2). Physics tells us that acceleration is the derivative of velocity:

$$
z^{\prime \prime}=(-1) \cdot \omega^{2} \cdot r \cdot \exp (i \omega t) \text {. }
$$

The leading vector of complex number $z^{\prime \prime}$ in physics is signified by $\boldsymbol{a}$, i.e. $\boldsymbol{a}=z^{\prime \prime}$. Since $-1=\exp (i \cdot \pi)$, formula (4) has the form:

$$
z^{\prime \prime}=\omega^{2} \cdot r \cdot \exp (i(\omega t+\pi)) \text {. }
$$

According to the formula above, the rotation of the versor of the linear velocity through the right angle corresponding with the direction of the rotation of the particle makes the versor of the vector of the linear acceleration of the particle, i.e. the equality sign $z^{\prime \prime}-$ sign $\mathrm{z}$ holds.

The second law of Newton states that the rate of change of momentum of the moving body is proportional to and in the same direction as the force acting on it, i.e. $\boldsymbol{F}=\boldsymbol{d}(m \boldsymbol{v}) / d t$, where $\boldsymbol{F}$ is the applied force of, $\boldsymbol{v}$ is the velocity of the body, and $m$ its mass. If the mass remains constant, $\boldsymbol{F}=m \boldsymbol{d} \boldsymbol{v} / d t$ or $\boldsymbol{F}=m \cdot \boldsymbol{a}$, i.e. $\boldsymbol{F}=m \cdot z^{\prime \prime}$, where

$$
m \cdot z^{\prime \prime}=m \cdot \omega^{2} \cdot r \cdot \exp (i(\omega t+\pi)) .
$$

If $m=1$, then the vector of linear acceleration is equal to the vector of the centripetal force. If the mass of the particle is not equal to 1, then the vector of the centripetal force has the same direction and sense as the vector of linear acceleration, but its length is not equal to it. The versors of the vectors of the acceleration and the centripetal force are equal, see Figure $3 .^{3}$

\footnotetext{
${ }^{3}$ If, for example, the particle is a weight rotating in a string, then the centripetal force is the tension of the string; if it is a ball in a roulette, then the centripetal force is the resilience force of the roulette board. During the motion in a circular path the centripetal force is balanced by an equal and opposite force, acting away from the centre of the circle, called centrifugal force. This force tightens the string and causes the pressure on the board of the roulette. A person is riding in a tram: on the curve of a tramline the passenger feels the pressure directed outside the curve. This is the effect of the acting centrifugal force. The passenger balances the pressure bending his/her trunk to the centre of the curve. Finally, the action of centrifugal force is balanced by the friction force between his/her body and the floor of the tram. A person driving a car puts on the free seat an apple. If the engine is turned off the apple rolls outside the bend. If the car is suddenly brought to a stop, the apple falls down to the floor of the car. If the speed of the car is increased, the apple rolls to the back of the car.
} 


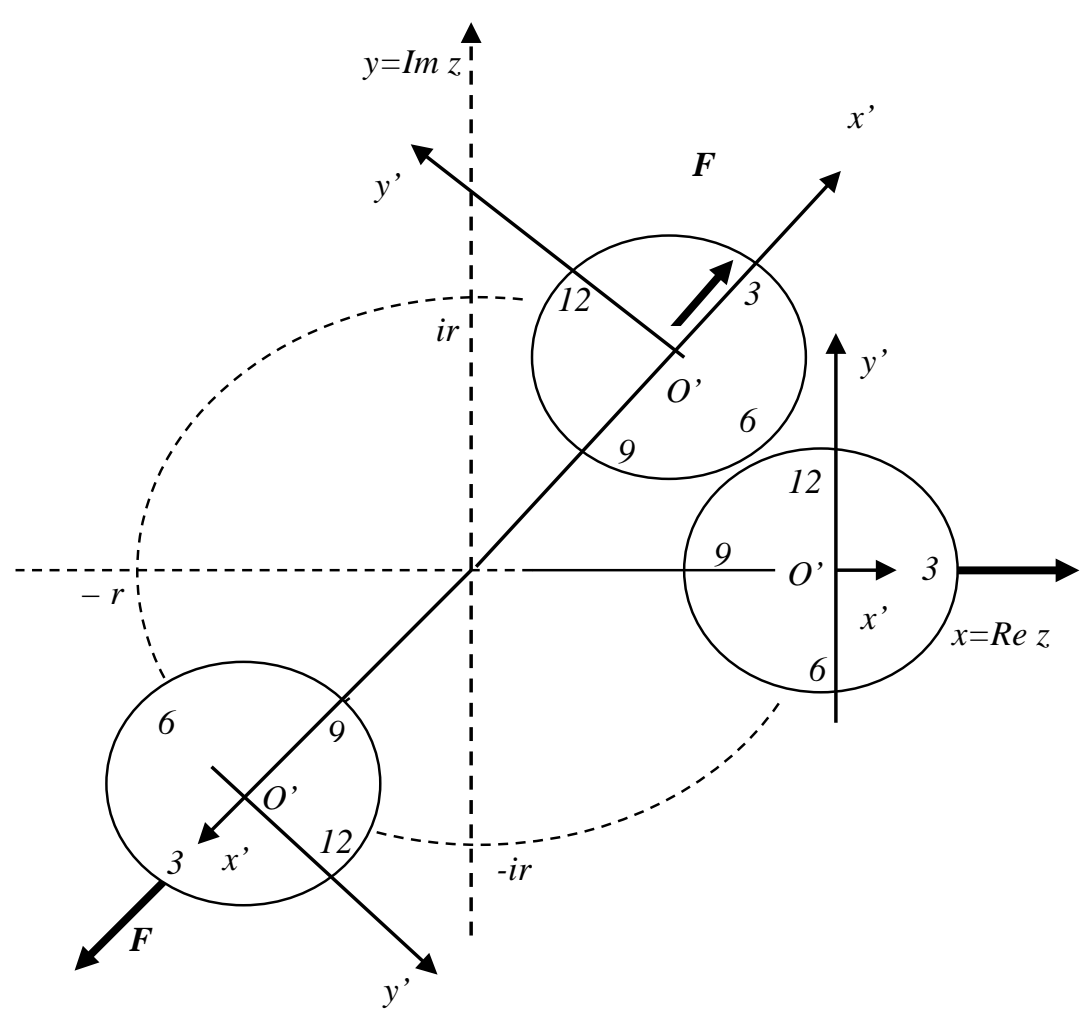

Fig. 4. Fictitious force - the centrifugal force

Source: own elaboration.

In Figure 4 point $(r, 0)$ is the origin of the new frame of reference $O^{\prime}, x^{\prime}$, $y^{\prime}$. In origin $O^{\prime}$ a clock is located in such a way that the positive $x^{\prime}$-axis is directed to the three and the positive $y$ '-axis to the twelve. The new system is rotating about the origin of the coordinate system $O, x, y$ in such a way that $O^{\prime}$ is rotating through the circle $x^{2}+y^{2}=r^{2}$, and origin $O$ has in the new system the coordinates $(-r, 0)$; Figure 4 shows three positions of the new system. In point $O^{\prime}$ along the line $O^{\prime}, O$ there is always the vector of linear acceleration, its sense is directed from $O^{\prime}$ to $O$, its length is equal to $r \cdot \omega^{2}$. It appears if on the point $O^{\prime}$ one is locating a particle of mass $m$. According to Newton's law, the vector has a length equal to $m \cdot r \cdot \omega^{2}$, its sense is opposite to the sense of the vector of linear acceleration, ${ }^{4}$ see Figure 4.

\footnotetext{
${ }^{4}$ If on the free seat in the car there is not any object, then in any point there is not any fictitious force. If one places an apple on the seat, the fictitious force will appear. The vector of acceleration always occurs.
} 
It is possible to introduce one more frame of reference $O$ ', $x$ ',,$y$ '’. Origin $O$ ' is the same point as point $O$. The system is rotating around point $O=O$ ' with the angular velocity $\omega$. In moment $t=0$ the positive $x$ ''-axis is the same as the $x$-axis, and the $y^{\prime \prime}$-axis is the same as the $y$-axis. The system $O, x, y$ is inertial and systems $O^{\prime}, x^{\prime}, y^{\prime}$ and $O$ ', $x$ ', $y$ ', are non-inertial.

The position of the particle in the inertial coordinate system $O, x, y$ is given by formula (1), in system $O^{\prime}, x^{\prime}, y^{\prime}$ the particle is always in origin $O^{\prime}$, in system $O$ ', $x$ ', $y$ '” it is located in point $(r, 0)$.

In Figure 4 the vector of fictitious force always shows three o'clock. This result is obtained in theory. It is possible to check it in practice, for which it is necessary to construct a face of a special clock.

\section{The construction and application of a fictitious force finding clock}

The face is not flat but it has the form of a crater or a funnel. The board of the face is similar to a roulette, see Figure 5 . In the centre of the crater one ought to put a little ball.

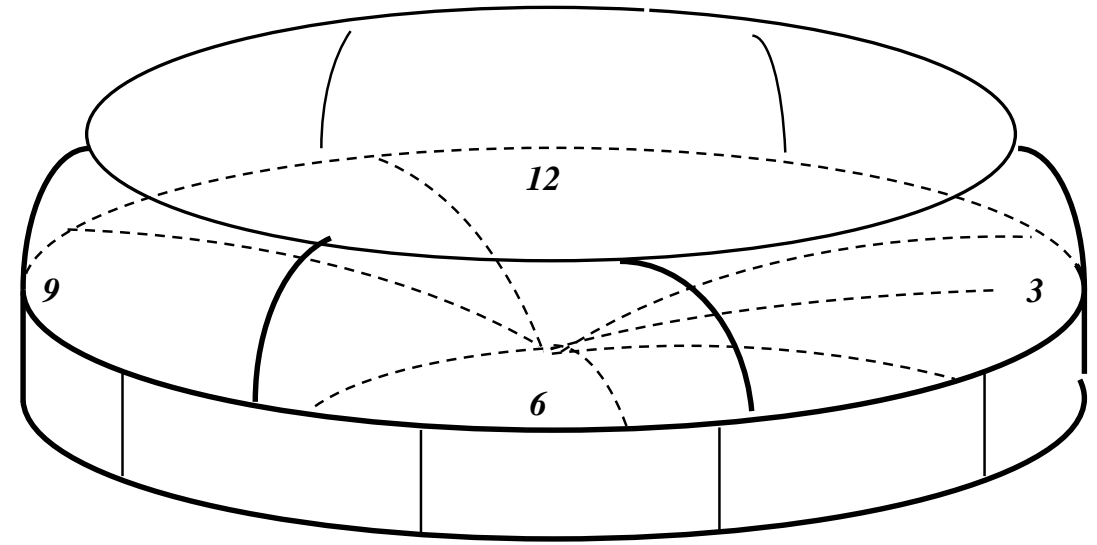

Fig. 5. The face of a fictitious force finding clock

Source: own elaboration. 
This clock should be located on a small merry-go-round as can be found in a playground. The centre of the merry-go-round and the hours nine and three lie in one and the same radius of the circle, it is certain that the centre of the clock and the centre of the merry-go-round are located in the two other points, see Figure 4.

One puts on the clock a little wooden or metal ball, and sets the merrygo-round in a rotational motion. During the motion the ball stays at the three o'clock. It shows the direction of action of the fictitious force. In this case it is the centrifugal force. The direction of the rotation has no influence on the direction of the centrifugal force. The merry-go-round can rotate clockwise or counter-clockwise, one way or another the ball stays in the same place three o'clock. The next point is to consider a non-inertial coordinate system that is not only rotating but also moving through a straight line.

\section{Uniform motion in a straight line in the rotating frame of reference}

Suppose that a particle is moving through the diameter of a circle, its linear velocity is constant, the circle is rotating about its centre at a constant angular velocity. ${ }^{5}$ Let the angular velocity be equal to $2 \pi$, i.e. at the unit of the time the disc is making one turn. At the same time the particle is moving from point $A(R, 0)$ to point $B(-R, 0)$, where $R$ is the radius of the circle.

In Figure 6 the time of one turn of the circle is equal to the time that the particle crosses through the diameter of the circle; the path of the particle divides the circle on two sides - with respect to the direction of the linear motion of the particle

Table 1 shows the position of the particle in some moments of the time in the inertial frame of reference using the polar coordinates.

Figure 7 shows the position of the particle according to Table 1 in the inertial frame of reference, the line of the points is the trajectory of the particle in this inertial frame of reference.

\footnotetext{
${ }^{5}$ It is easy to assume these conditions. The next stage is showing in an experiment in what way that motion is possible in practice.
} 


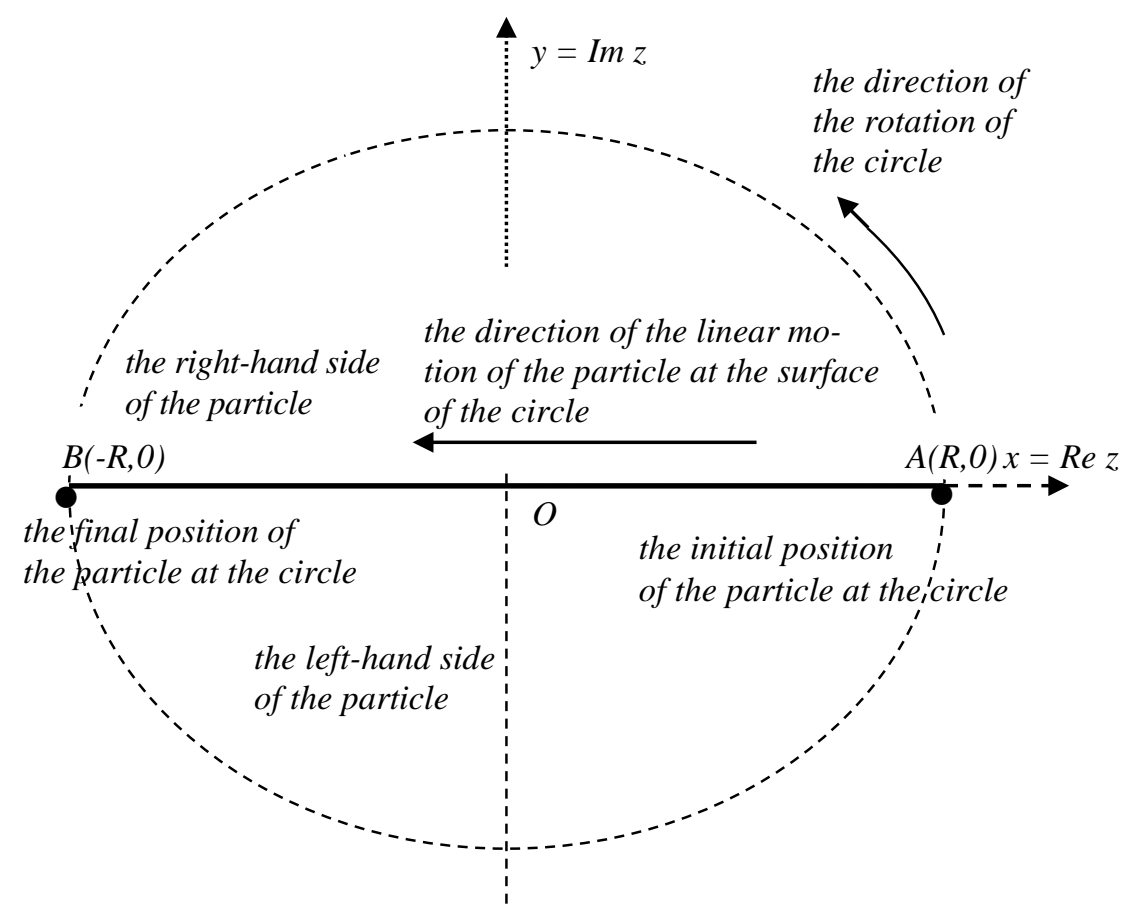

Fig. 6. The rotational motion of the circle and the linear motion of the particle on the surface of the circle

Source: own elaboration.

Table 1 . The position of the particle in certain moments of time

\begin{tabular}{|c|c|c|c|c|c|c|c|c|c|}
\hline time & 0 & $1 / 12$ & $1 / 8$ & $1 / 6$ & $1 / 4$ & $1 / 3$ & $3 / 8$ & $5 / 12$ & $1 / 2$ \\
\hline angle & $\pi$ & $\pi / 6$ & $\pi / 4$ & $\pi / 3$ & $\pi / 2$ & $(2 / 3) \pi$ & $(3 / 4) \pi$ & $(5 / 6) \pi$ & none \\
\hline radius & $R$ & $(5 / 6) R$ & $(3 / 4) R$ & $(2 / 3) R$ & $R / 2$ & $R / 3$ & $R / 4$ & $R / 6$ & 0 \\
\hline point & $A_{0}=A$ & $A_{1}$ & $A_{2}$ & $A_{3}$ & $A_{4}$ & $A_{6}$ & $A_{6}$ & $A_{7}$ & $A_{8}=0$ \\
\hline
\end{tabular}

\begin{tabular}{|c|c|c|c|c|c|c|c|c|c|}
\hline time & $1 / 2$ & $7 / 12$ & $5 / 8$ & $2 / 3$ & $3 / 4$ & $5 / 6$ & $7 / 8$ & $11 / 12$ & 1 \\
\hline angle & none & $\pi / 6$ & $(1 / 4) \pi$ & $\pi / 3$ & $\pi / 2$ & $(2 / 3) \pi$ & $(3 / 4) \pi$ & $(5 / 6) \pi$ & $\pi$ \\
\hline radius & 0 & $R / 6$ & $R / 4$ & $R / 3$ & $R / 2$ & $(2 / 3) R$ & $(3 / 4) R$ & $(5 / 6) R$ & $R$ \\
\hline point & $A_{8}=0$ & $A_{9}$ & $A_{10}$ & $A_{11}$ & $A_{12}=A_{4}$ & $A_{13}$ & $A_{14}$ & $A_{15}$ & $A_{16}=B$ \\
\hline
\end{tabular}

Source: own elaboration. 


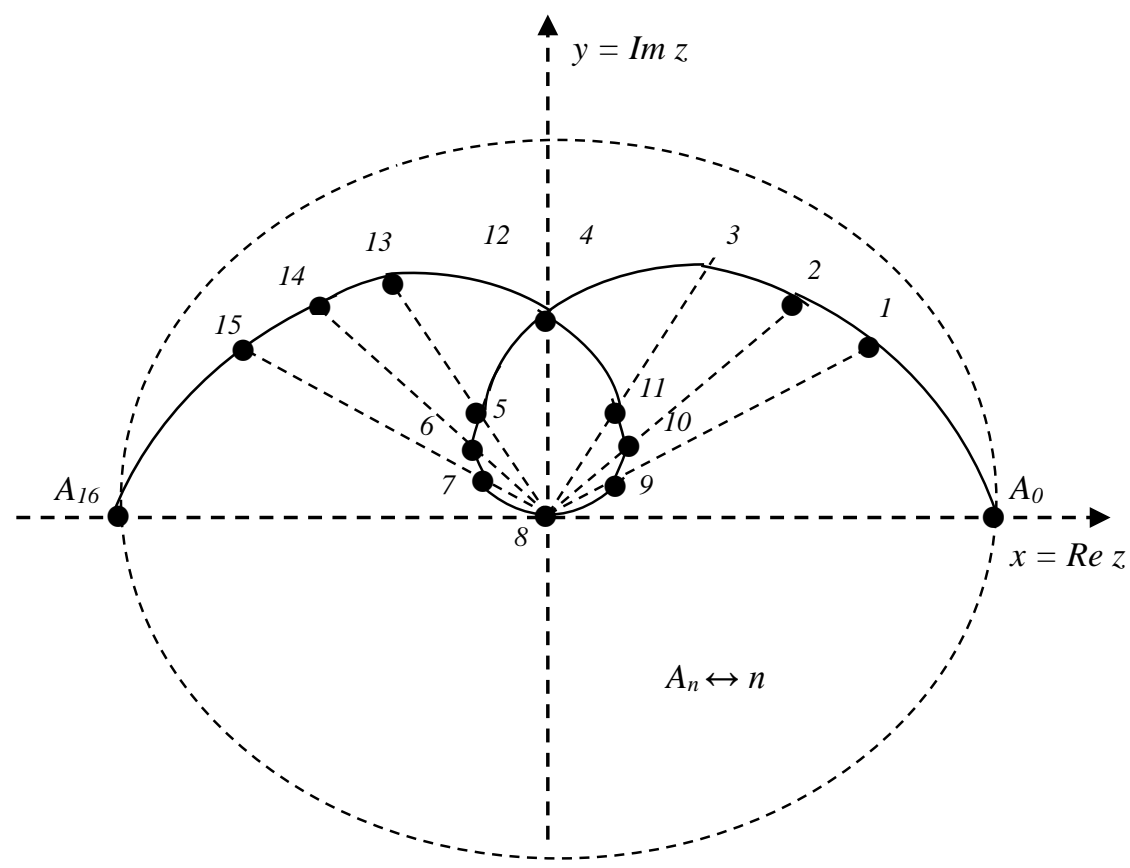

Fig. 7. Trajectory of the particle in the inertial frame of reference

Source: own elaboration.

The equation of the trajectory is equal to

$$
z=R \cdot(1-2 t) \cdot \exp (i 2 \pi t),(7) \text { written } \boldsymbol{r}=z \text {, where }
$$

$\boldsymbol{r}$ is the leading vector of the position of the particle. The first derivative of function (7) is the vector of the velocity of the particle in the inertial frame of reference, the second derivative is the vector of the acceleration:

i.e.

$$
z^{\prime}=-2 R \cdot \exp (i 2 \pi t)+i \cdot 2 \pi R \cdot(1-2 t) \cdot \exp (i 2 \pi t),
$$

$$
z^{\prime}=-2 R \cdot \exp (i 2 \pi t)+2 \pi \cdot R \cdot(1-2 t) \cdot \exp \left(i \cdot\left(2 \pi t+\frac{\pi}{2}\right)\right)
$$

by the formulae above $\boldsymbol{v}=z^{\prime}$, and $\boldsymbol{v}=z$ '’. Since

$$
\exp (i 2 \pi t)=\cos (2 \pi t)+i \sin (2 \pi t)
$$

the vector

$$
v_{r}=-2 R \cdot(\cos 2 \pi \cdot t, \sin 2 \pi \cdot t)
$$


is directed according to the vector of the position of the particle but its sense is contrary to it. Its length is equal to $2 R$ according to the linear velocity of the particle on the linear motion through the diameter of the circle. Vector $v_{r}$ is parallel to the vector $\overrightarrow{A_{t}, B_{t}}$, where points $A_{t}$ and $B_{t}$ are the positions of points $A_{0}$ and $B_{0}$, if the circle is turning through angle $2 \pi \cdot t$, i.e. in the moment $t$ of time, see Figure 8, i.e.

$$
A_{t}=(R \cdot \cos 2 \pi \cdot t, \quad R \cdot \sin 2 \pi \cdot t)
$$

and

$$
B_{t}=(-R \cdot \cos 2 \pi \cdot t,-R \cdot \sin 2 \pi \cdot t) .
$$

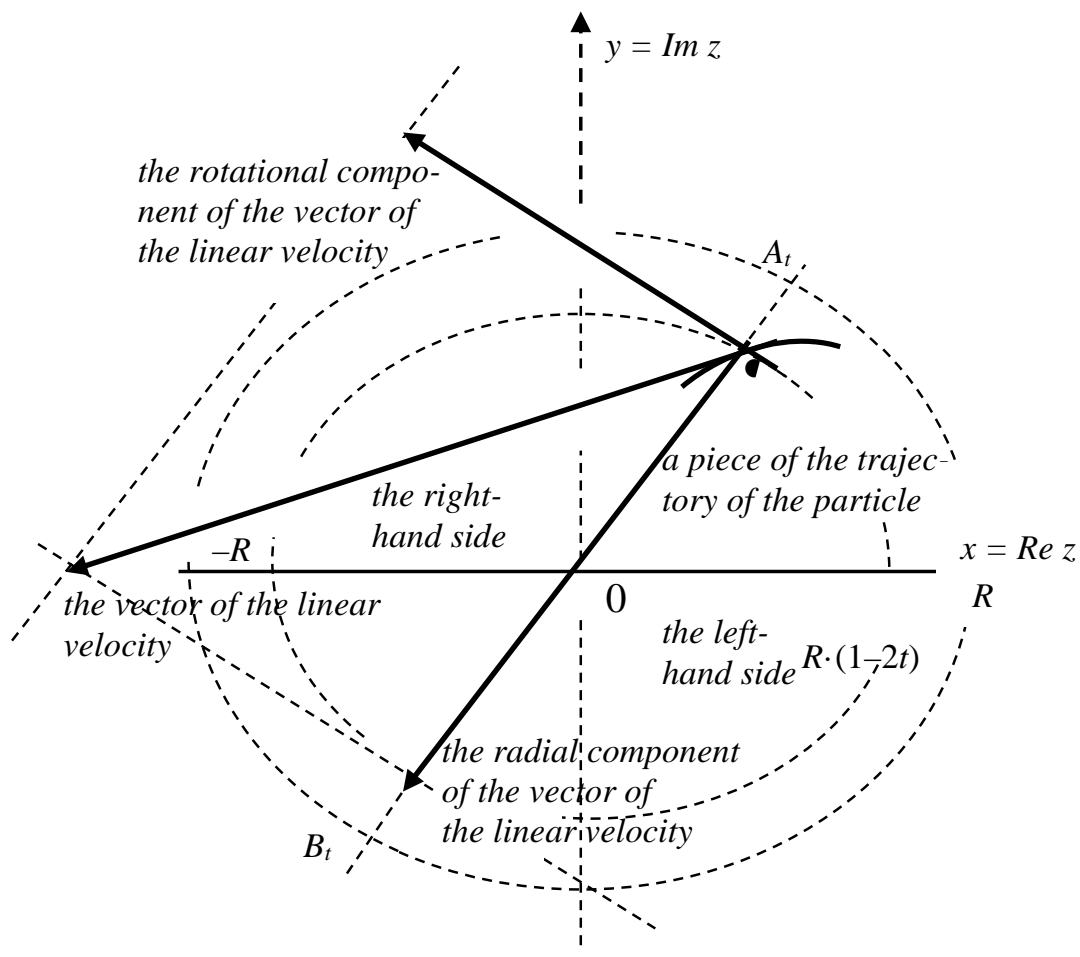

Fig. 8. Decomposition of the vector of linear velocity of the particle in the inertial frame of reference; in the point of the trajectory for the moment of time $t<1 / 4$

Source: own elaboration. 
The rotational component of the vector of the linear velocity is perpendicular to the leading vector of the particle, the length of it is equal to $2 \pi R \cdot(1-2 t)$; it is equal to the vector of the velocity of the point of the circle in which the particle is situated at moment $t$. This component measures the velocity with respect to the rotation of the disc. In Figure 8 the moment $t$ of time belongs to interval $(0,1 / 4)$. The linear speed of the particle ${ }^{6}$ with respect to the inertial frame of reference is changeable. This is the function of variable $t$ :

$$
v=2 R \cdot \sqrt{1+\pi^{2} \cdot(1-2 t)^{2}} .
$$

The smallest value of it is equal to $2 R$, for $t=1 / 2$; the particle at the moment is located in the centre of the circle. The greatest value is equal to $2 R \cdot \sqrt{1+\pi^{2}}$, for $t=0$ and $t=1$. The speed is a decreasing function in interval $(0,1 / 2)$ and an increasing in $(1 / 2,1)$, see Figure 7 . The decomposition of the vector of the linear velocity of the particle with respect to the inertial frame of reference is presented in Figure 8.

To find the linear acceleration of the particle in the inertial frame of reference it is necessary to calculate the second derivative of the function of the position of the particle, i.e.to calculate the derivative of function (8):

$$
\begin{aligned}
z^{\prime \prime}= & -2 R \cdot i \cdot 2 \pi \cdot \exp (2 \pi i t)- \\
& 2 \cdot i \cdot 2 \pi R \cdot \exp (2 \pi i t)-2 \pi \cdot 2 \pi R \cdot(1-2 t) \cdot \exp (2 \pi i t),
\end{aligned}
$$

the result would be given in a more simple form:

$$
z^{\prime \prime}=-8 \pi R \cdot i \cdot \exp (i 2 \pi t)-4 \pi^{2} R \cdot(1-2 t) \cdot \exp (i 2 \pi t),
$$

i.e.

$$
z^{\prime \prime}=-8 \pi R \cdot \exp \left(i\left(2 \pi t+\frac{\pi}{2}\right)\right)-4 \pi^{2} R \cdot(1-2 t) \cdot \exp (i 2 \pi t) .
$$

Since $\boldsymbol{a}=\mathrm{z}$ ", the vector of acceleration has two components: centripetal acceleration $\boldsymbol{a}_{c p}$ :

$$
\boldsymbol{a}_{c p}=-4 \pi^{2} R \cdot(1-2 t) \cdot \exp (i \quad 2 \pi t)
$$

and Coriolis acceleration ${ }^{7} \boldsymbol{a}_{C}$ :

$$
\boldsymbol{a}_{C}=-8 \pi R \cdot \exp \left(i\left(2 \pi t+\frac{\pi}{2}\right)\right) .
$$

\footnotetext{
${ }^{6}$ The velocity is a vector, the speed is the length of this vector, it is a scalar.

7 [Resnick, Halliday 1980, p. 773].
} 


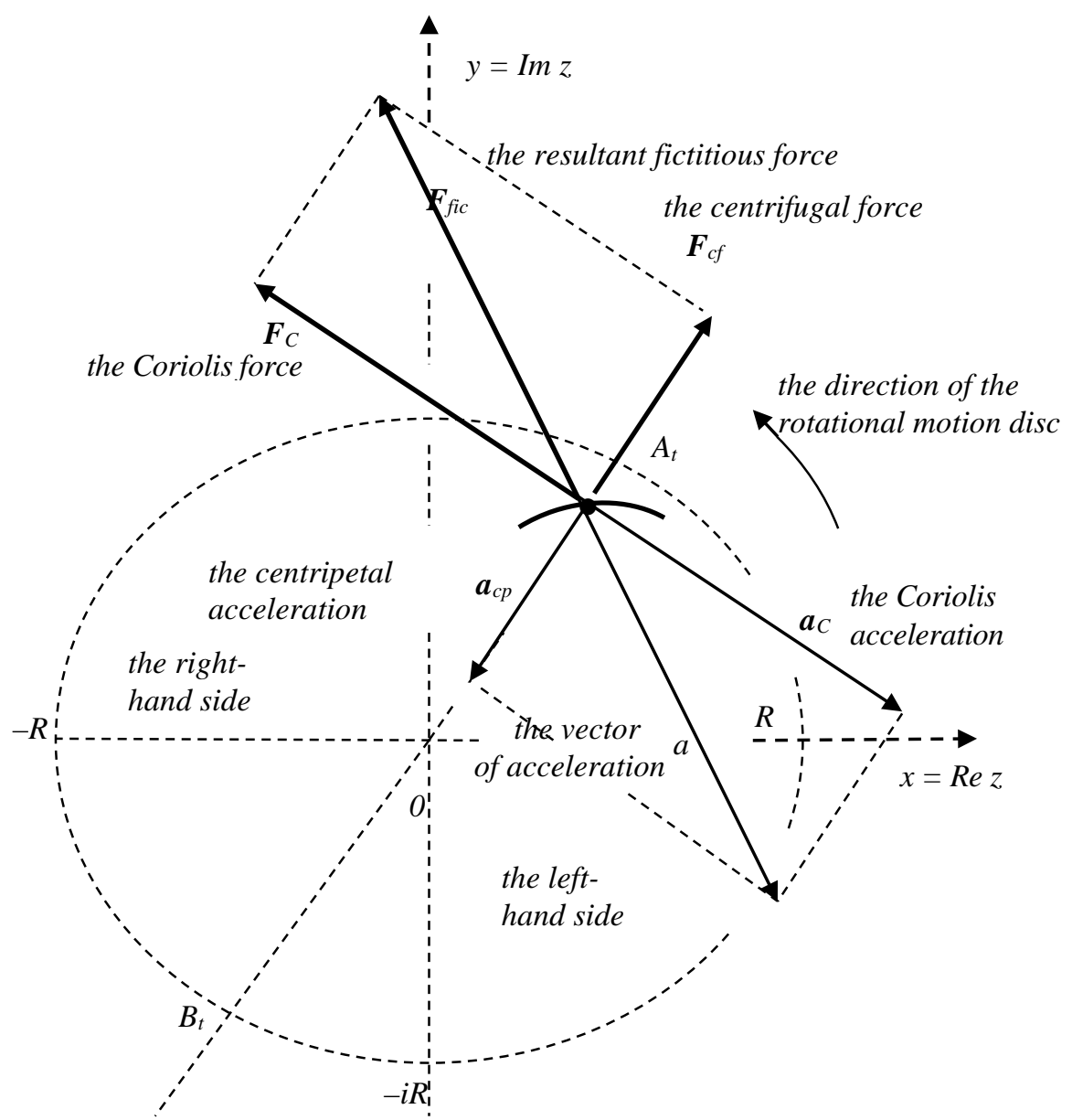

Fig. 9. The acceleration vectors - in the inertial frame of reference and the vectors of the fictitious forces - in the rotating frame of reference

Source: own elaboration.

If the mass of the particle is equal to the unit, then $\mathbf{F}_{\mathrm{cf}}=-\mathbf{a}_{\mathrm{cp}}$ and $\mathbf{F}_{\mathrm{C}}=-\mathbf{a}_{\mathrm{C}}$ and $\mathbf{F}_{\text {fic }}=-\mathbf{a}$. The direction of the linear motion of the particle on the surface of the disc is running from $A_{s}$ to $B_{s}$. From the direction is defined the right and left-hand side.

The coordinate system $O$ ', $x$ ', $y^{\prime \prime}$ ' has the same origin as the inertial coordinate system $O, x, y$, i.e. $O=O$ '’. System $O$ ', $x$ ', $y$ ', is rotating about the origin at angular velocity $2 \pi$, i.e. the disc is rotating through $360^{\circ}$ during the unit of the time. In this frame of reference the particle has the location: 
$x^{\prime \prime}=R \cdot(1-2 t)$, and $y^{\prime \prime}=O$, where $t \in(0,1)$. Two fictitious forces act on the particle: centrifugal force $\boldsymbol{F}_{c f}$ and Coriolis force $\boldsymbol{F}_{C}$; the resultant fictitious force $\boldsymbol{F}_{\text {fic }}$ that acts on the particle at a particular moment of time is the sum of centrifugal force $\boldsymbol{F}_{\boldsymbol{c} \boldsymbol{f}}$ and Coriolis force $\boldsymbol{F}_{\boldsymbol{C}}{ }^{8}$ similarly the acceleration of the particle with respect to the inertial frame of reference, symbol $\boldsymbol{a}$, has two components: centrifugal acceleration $\boldsymbol{a}_{\boldsymbol{c} p}$ and Coriolis acceleration $\boldsymbol{a}_{\boldsymbol{C}}$ at the same moment of the time.
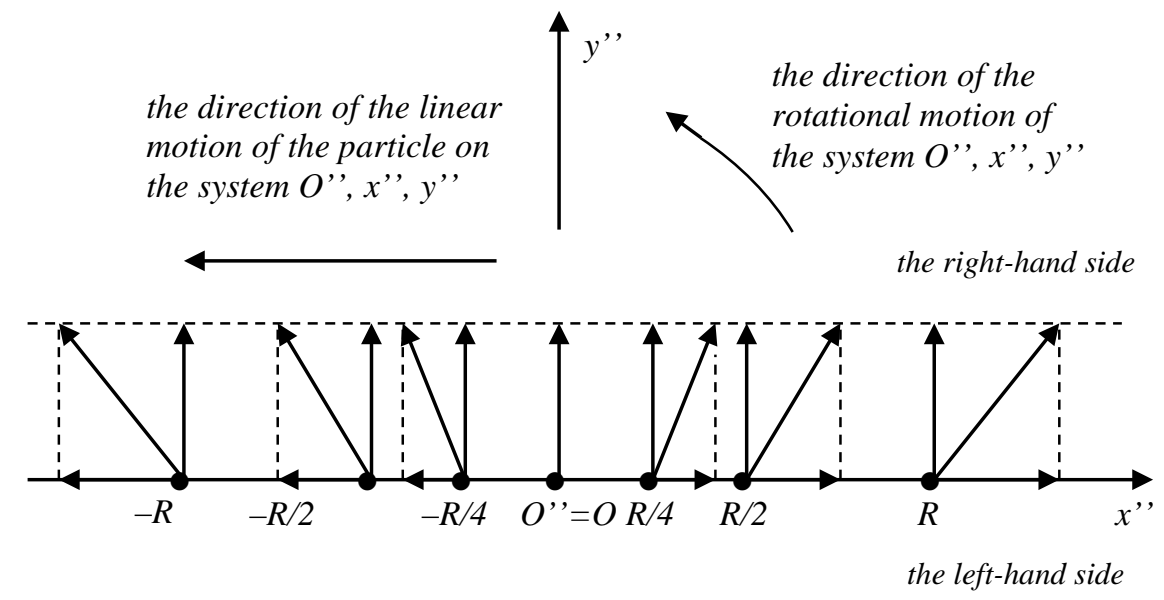

Fig. 10. The fictitious forces that act at the particle that is moving on the linear path with the constant velocity in the rotating frame of reference

Source: own elaboration.

Each horizontal vector is the representation of the centrifugal force dependent on the $x$ ''-axis. Each vertical vector is a representation of the Coriolis force, it is one and the same in the rotating system $O$ ', $x$ ', $y$ '” through the path of the particle. The sum of the vertical and horizontal vector is the vector of the resultant fictitious force in the rotating system $O$ ', $x$ ', $y$ '”.

The formulae of the fictitious forces: the centrifugal force and the Coriolis force are similar to the formulae of the Coriolis acceleration and centripetal acceleration. To find the force it is necessary to write the sign of minus and the mass of the particle to the formula of acceleration:

$$
\boldsymbol{F}_{c f}=m \cdot 4 \pi^{2} R \cdot(1-2 t) \cdot \exp (i \quad 2 \pi t)
$$

\footnotetext{
${ }^{8}$ See Figures 9 and 10.
} 
and

$$
\boldsymbol{F}_{C}=m \cdot 8 \pi R \cdot \exp \left(i\left(2 \pi t+\frac{\pi}{2}\right)\right),
$$

see (14) and (15). The formulae are the same as in the textbooks. ${ }^{9}$ Centrifugal force $\boldsymbol{F}_{\boldsymbol{c} \boldsymbol{f}}=m \cdot \omega^{2} \cdot \boldsymbol{r}$ and Coriolis force $\boldsymbol{F}_{\boldsymbol{C}}=2 m\left(\boldsymbol{v}_{\boldsymbol{1}} \times \boldsymbol{\omega}\right)$, where $\boldsymbol{v}_{\mathbf{1}}$ is the vector of linear velocity on the rotating frame of reference and $\omega$ is the vector of angular velocity of it.

It is possible to count the absolute value of the tangent of the angle that is made by the resultant fictitious force and the Coriolis force:

$$
\operatorname{tg} \beta(t)= \pm \frac{\left|F_{c f}\right|}{\left|F_{C}\right|}=\frac{m \cdot 4 \pi^{2} R \cdot(1-2 t)}{2 m \cdot 4 \pi R}=-\frac{\pi \cdot(1-2 t)}{2} .
$$

The sign of the tangent depends on the direction of the linear motion of the particle at the surface of the turning disc and whether the disc is turned clockwise or counter-clockwise, see Figure 12. The absolute value of the tangent is presented in Table 2:

Table 2. The direction of the fictitious force in the time

\begin{tabular}{|c|c|c|c|c|c|c|c|c|c|}
\hline the time & 0 & $1 / 12$ & $1 / 8$ & $1 / 6$ & $1 / 4$ & $1 / 3$ & $3 / 8$ & $5 / 12$ & $1 / 2$ \\
\hline $\tan \beta(t)$ & -1.57 & -1.31 & -1.18 & -1.05 & -0.78 & -0.52 & -0.39 & -0.26 & 0 \\
\hline$\beta(t)$ & $-57^{\circ} 30^{\prime}$ & $-52^{\circ} 35^{\prime}$ & $-49^{\circ} 45^{\prime}$ & $-46^{\circ} 25^{\prime}$ & $-37^{\circ} 55^{\prime}$ & $-27^{\circ} 30^{\prime}$ & $-21^{\circ} 20^{\prime}$ & $-14^{\circ} 35^{\prime}$ & $0^{\circ}$ \\
\hline$h(t)$ & $1: 55$ & $1: 45$ & $1: 40$ & $1: 33$ & $1: 16$ & $0: 55$ & $0: 43$ & $0: 29$ & $0: 00$ \\
\hline
\end{tabular}

\begin{tabular}{|c|c|c|c|c|c|c|c|c|c|}
\hline the time & $1 / 2$ & $7 / 12$ & $5 / 8$ & $2 / 3$ & $3 / 4$ & $5 / 6$ & $7 / 8$ & $11 / 12$ & 1 \\
\hline $\tan \beta(t)$ & 0 & 0.26 & 0.39 & 0.52 & 0.78 & 1.05 & 1.18 & 1.31 & 1.57 \\
\hline$\beta(t)$ & $0^{\circ}$ & $14^{\circ} 35^{\prime}$ & $21^{\circ} 20^{\prime}$ & $27^{\circ} 30^{\prime}$ & $37^{\circ} 55^{\prime}$ & $46^{\circ} 25^{\prime}$ & $49^{\circ} 45^{\prime}$ & $52^{\circ} 35^{\prime}$ & $57^{\circ} 30^{\prime}$ \\
\hline$h(t)$ & $0: 00$ & $11: 31$ & $11: 17$ & $11: 05$ & $10: 44$ & $10: 27$ & $10: 20$ & $10: 15$ & $10: 05$ \\
\hline
\end{tabular}

Source: own elaboration.

Now, in the position of the particle is located the origin of the frame of reference $O^{\prime}, x^{\prime}, y^{\prime}$ in such a way that the $x^{\prime}$-axis is determined by the origin of the inertial frame of reference $O, x, y$ and the position of the particle in

\footnotetext{
${ }^{9}$ See: [Feynman, Leighton, Sands 1974, p. 290; Bobrowski 1998, p. 65].
} 
moment $t=0$, i.e. by point $(0, R)$ in system $O, x, y$, the $y^{\prime}$-axis is perpendicular to the $x^{\prime}$-axis and simultaneously the angle between the positive $x^{\prime}$-axis and the positive $y^{\prime}$-axis is counter-clockwise. Each point of system $O^{\prime}, x^{\prime}, y^{\prime}$ is moving at a linear path in respect to system $O$ ',,$x$ ', $y$ ', origin $O$ ' has the same position in respect to the inertial frame of reference $O, x, y$ as that of particle (7).

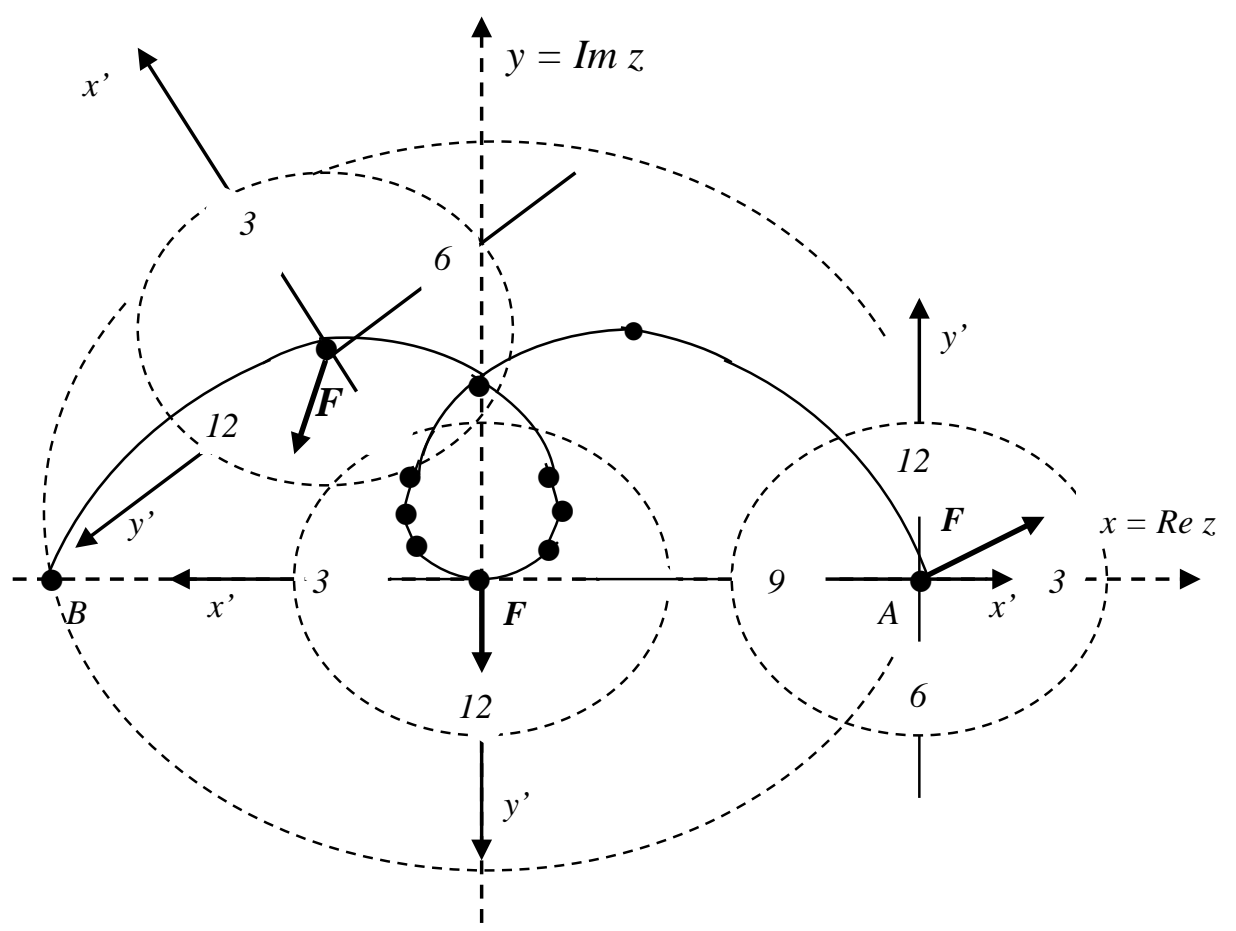

Fig. 11. The resultant fictitious forces

Source: own elaboration.

In the origin of system $O^{\prime}, x^{\prime}, y^{\prime}$ there is located a clock, in such a way that the $y$ '-axis shows 12 o'clock and the $x^{\prime}$-axis, 3 o'clock. This way the Coriolis force that acts on the particle which is located in origin $O$ ' shows 12 o'clock and has always one and the same value $8 \mathrm{~m} \pi \mathrm{R}$; the centrifugal force is directed through the $x$ '-axis and has the sense compatible to the positive of it for $t \in(0,1 / 2)$, to the negative for $t \in(1 / 2,1)$, for $t=1 / 2$ origin $O^{\prime}$ has the same position in respect to the inertial frame of reference as that of its origin 
$O$, consequently the centrifugal force equals zero, see Figure $11 .^{10}$ The value of the centrifugal force in respect to the time is equal to $4 m \pi R \cdot(1-2 t)$, in the linear frame of reference $O^{\prime}, x^{\prime}$ it would be positive or negative. In Figure 11 the resultant fictitious force is shown at three moments of the time: $t=0$, $t=1 / 2, t=5 / 6{ }^{11}$

In the inertial frame of reference $O, x, y$, system $O^{\prime}, x^{\prime}, y^{\prime}$ has the location that is changeable in time. The positive $x^{\prime}$-axis always shows 3 o'clock and the y'-axis, 12 o'clock.

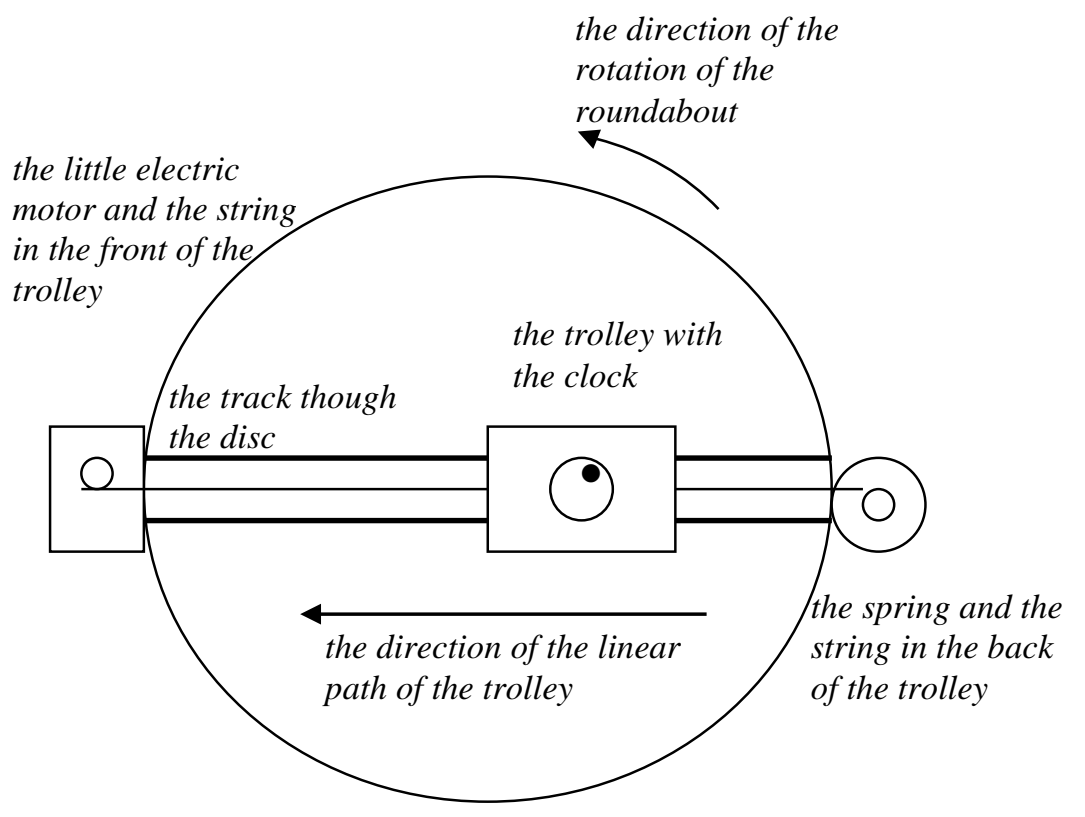

Fig. 12. The trolley with the clock in the track on the surface of the merry-go-round Source: own elaboration.

\footnotetext{
${ }^{10}$ If the direction of the rotation of the disc is couter-clockwise, then the Coriolis force and the resultant fictitious force is directed on the right-hand side with respect to the direction of the particle; similarly these forces are directed on the left-hand side if the rotation is directed clockwise. In harmony to this conclusion on the northern hemisphere rivers destroy the right-hand edges southern hemisphere the left-hand edges, see Bobrowski [1998], p. 65.

${ }^{11}$ See: Figure 7 and Tables 1 and 2.
} 
It is possible to verify in practice the results found in theory. To this end it would be put through the centre of the merry-go-round ${ }^{12}$ a track for a little wagon; on this trolley is located the clock, see Figure 5 . The roundabout would be moved by an electric motor or simply by hand. This is need to force the linear motion of the trolley along the track. In the front and back of it would be fastened with a rope. The string in the front would be pulled by a little electric motor that is situated in the edge of the disc. The trolley is held back by the second string fastened in the back of the wagon - a spring located in the opposite edge of the trolley is pulling it back. The spring is needed to neutralize the centrifugal force which occurs when the trolley is passing the centre of the roundabout. In this way it would get a motion simultaneously rotational and linear. In Figure 12 a view of this situation is presented from above.

The ball of the clock shows the direction in which acts the resultant fictitious force. It is possible to film the experiment from above and in this way to vet the theory results that are presented in Table 2 .

There is a simple method to would look for the direction in which the Coriolis force acts. It requires finding in a playground a merry-go-round with an axle-tree in its centre ${ }^{13}$. On the surface of the roundabout it is necessary to place two parallel planks as in Figure 13; round the axle a string is wound, on the end of a block is tied. If the carousel is rotating, the string is winding round the axle and the block is shifting from the edge of the disc to the centre of it. The block is moving through the edge of one of these two planks. The block is pressing onto the edge by the Coriolis force. The direction of the force depends on the direction of the rotation of the disc, see Figure 13. If the block is situated close to the axle of the roundabout without the string, then during the rotating motion of the disc the block is pushed aside from the centre of the merry-go-round to its edge by the centripetal force. And again the Coriolis force is pushing aside the block to the edge of one of the two planks. The Coriolis force acts only on a body which is moving in some rotating frame of reference. The direction of the Coriolis force depends on the direction of the rotation of the disc, and the direction of the linear motion of the body is presented in Figure 13.

\footnotetext{
12 It is need to find a roundabout without an axis in its center. The handrail must be situated on the edge of it.

${ }^{13}$ In this model of the merry-go-round, three handrails are situated evenly like in the Mercedes symbol.
} 


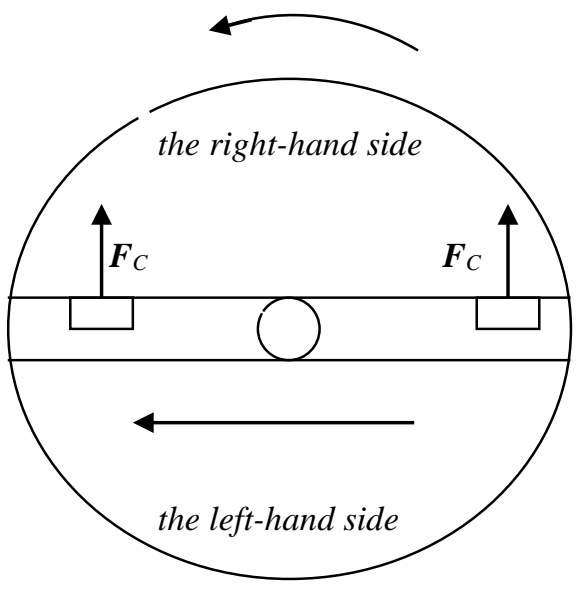

Fig. $13 a$

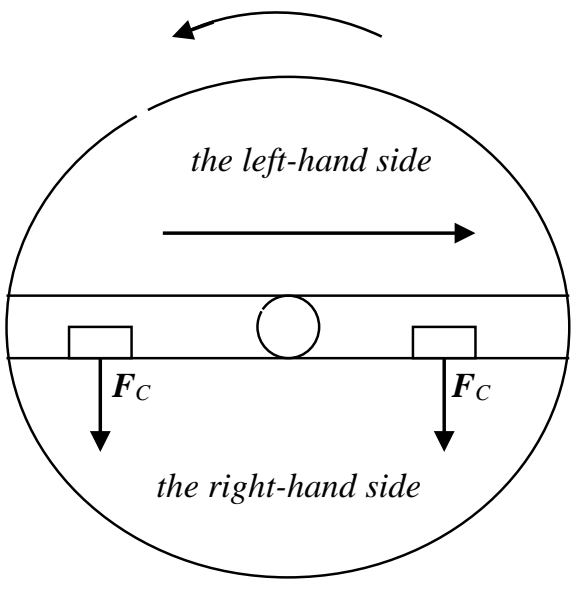

Fig. $13 c$

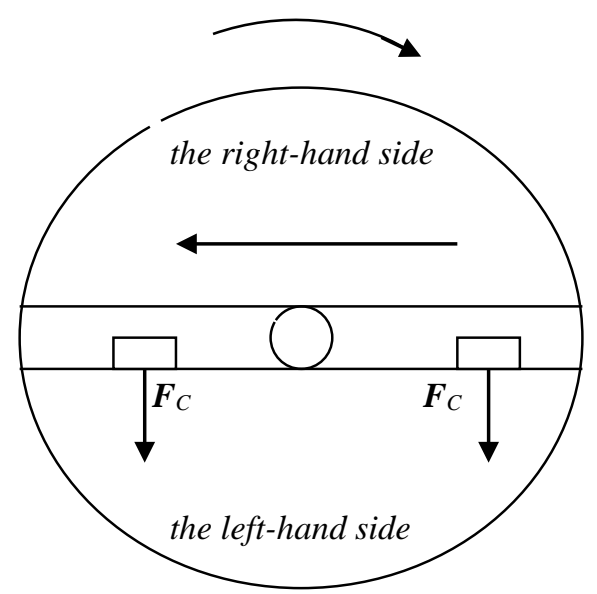

Fig. $13 b$

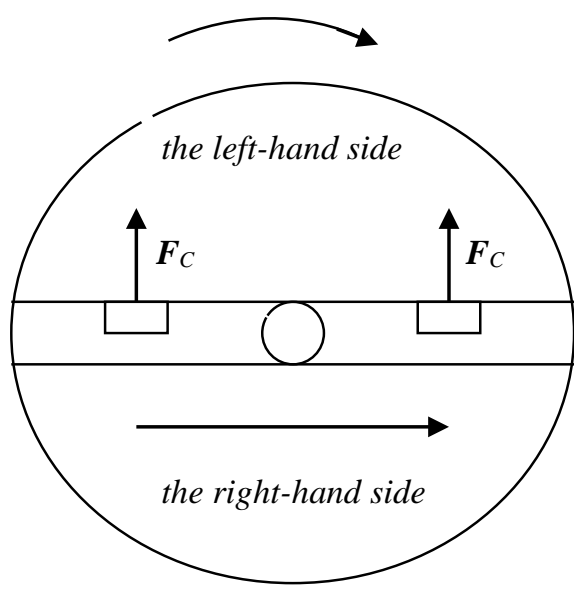

Fig. $13 d$

Fig. 13. The direction of the action of the Coriolis force Source: own elaboration.

Figure 13 would help to understand why rivers in the northern hemisphere are pushing at the right-side bank: Figures $13 a$ and $13 c$, and at the leftside bank in the southern hemisphere: Figures $13 b$ and $13 d$. 


\section{Coriolis power plant}

It is known that demand for energy is huge and constantly growing. Looking for new sources of energy is nowadays a necessity. An inexhaustible source of energy is the rotational motion of the Earth, but an idea of how to do it is needed. There is a simple means to build a power plant using the energy of the rotation of the Earth, making use of the Coriolis force which acts on a stream of water.

It does not need to be a big river step. Figure 14 shows a system of containers - tubs. In the outlet of each tub a turbine is placed.

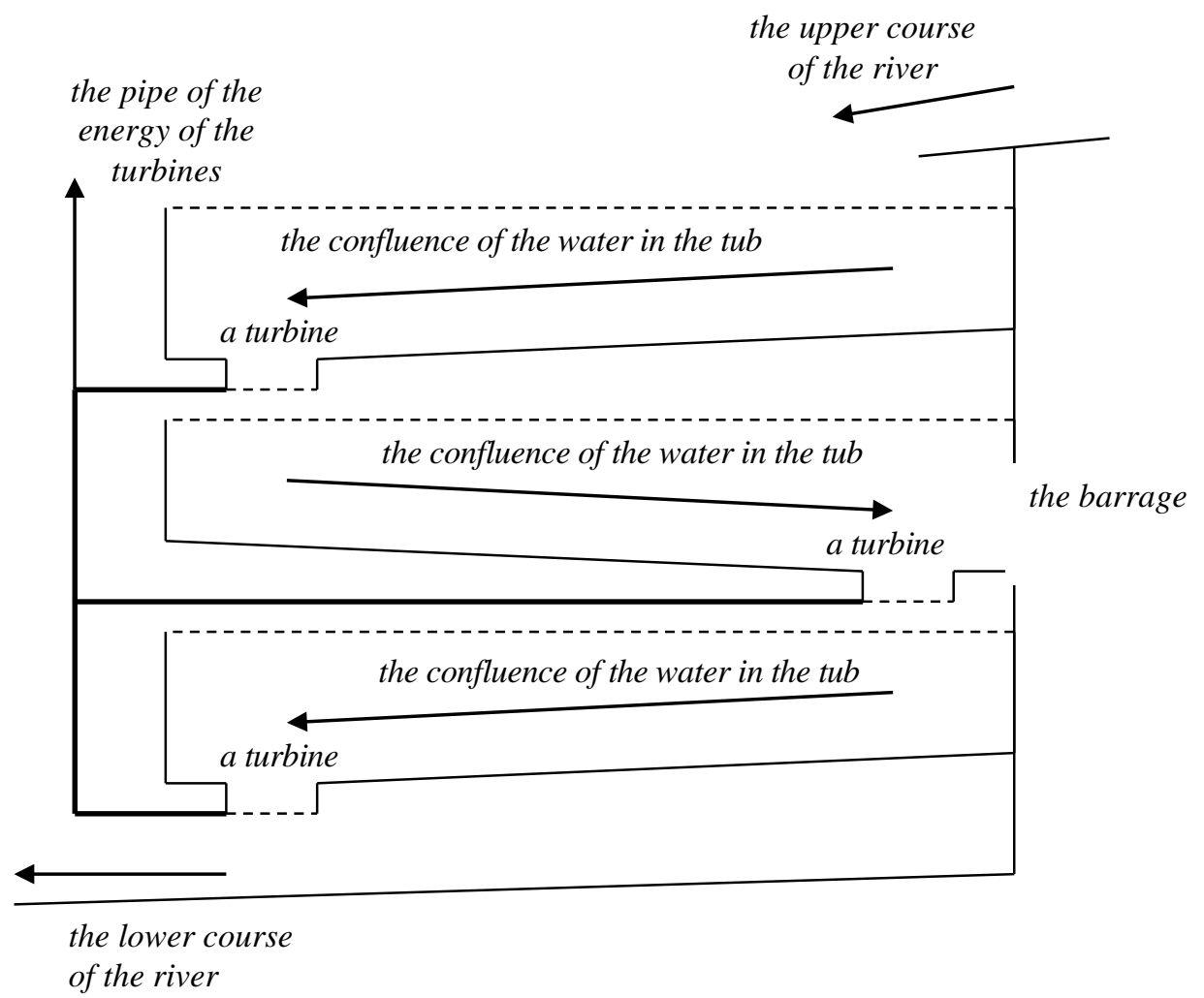

Fig. 14. The scheme of the Coriolis power plant

Source: own elaboration. 
The water flows through each tub. Because of it and because of the rotation of the Earth, the Coriolis force acts on the flowing water. In the outflow, the water causes the rotation of the turbine and flows from one to the next tub. On the way each turbine produces the electric energy which it is needed to collect and to send into the energy system.

\section{Application in the economy}

In theory of economics there is a trend which interprets the physical laws and equations in the economy. It is a question whether there can be some interpretations in the economy of the fictitious forces: Coriolis and centrifugal.

\section{Bibliography}

Bobrowski C. (1998). Fizyka - krótki kurs. Wydawnictwa Naukowo-Techniczne. Warszawa. Clapham C. (1996). The Concise Oxford Dictionary of Mathematics. Oxford University Press. Feynman R.P., Leighton R.B., Sands M. (1974). Feynmana wykłady z fizyki. PWN. Warszawa. Oxford (2015). Dictionary of Physics. Oxford University Press.

Resnick R., Halliday D. (1980). Fizyka. PWN. Warszawa. 